\title{
REDUCTION OF SECOND ORDER LINEAR DYNAMICAL SYSTEMS, WITH LARGE DISSIPATION, BY STATE VARIABLE TRANSFORMATIONS
}

\author{
R. B. LEIPNIK
}

(Received March 1989; revised November 1989)

\begin{abstract}
Linear dynamical systems of the Rayleigh form $M \ddot{q}+C \dot{q}+K q=f$ are transformed by linear state variable transformations $w=A \dot{q}+B q$, where $A$ and $B$ are chosen to simplify analysis and reduce computing time. In particular, $A$ is essentially a square root of $M$, and $B$ is a Lyapunov quotient of $C$ by $A$. Neither $K$ nor $C$ is required to be symmetric, nor is $C$ small. The resulting state-space systems are analysed by factorisation of the evolution matrices into reducible factors. Eigenvectors and eigenvalues are determined by these factors. Conditions for further simplification are derived in terms of Kronecker determinants. These results are compared with classical reductions of Rayleigh, Duncan, and Caughey, which are reviewed at the outset.
\end{abstract}

\section{Introduction}

Lagrangian systems with finite degrees of freedom are important in astronomy, particle mechanics, and lumped system electromagnetism. When nonlinear, they are usually treated by perturbations about steady or periodic states, although a few systems have been completely integrable (Hamel [8], Lax [10]). The resulting perturbation equations are generally second-order linear systems of the damped Rayleigh type although sometimes they are quadratic systems, which can display limit cycles or chaos in some parameter regions. When the linear systems are of low order or the dissipation terms are relatively small, the computational problems are manageable. However, high-order systems with large dissipation are computationally difficult, although the theory is well enough understood. Rayleigh systems also appear

\footnotetext{
${ }^{1}$ Department of Mathematics, University of California, Santa Barbara, U.S.A.

(C) Copyright Australian Mathematical Society 1990, Serial-fee code 0334-2700/90
} 
in finite-element approximations to dynamical continuum problems, except that the number of degrees of freedom varies with the number and type of "elements" chosen.

A Rayleigh system is characterised by three matrices $M, C$, and $K$, which may be time-dependent. The mass matrix $M$ is symmetric and positive definite. The Rayleigh matrix $C=C_{1}+C_{2}$ and Hooke matrix $K=K_{1}+K_{2}$ where $C_{1}, K_{1}$ are symmetric (or Hermitian in electromagnetic problems) and $C_{2}, K_{2}$ are anti-symmetric (or anti-Hermitian). $C_{1}$ represents dissipation, $C_{2}$ represents gyroscopic or magnetic forces, $K_{1}$ represents conservative restoring forces, and $K_{2}$ represents non-conservative restoring (circulatory) forces. Most treatments of dissipation in high order systems assume that $C_{1}$ is small and that $C_{2}=K_{2}=0$.

A concise review of the material can be found in Frank and von Mises [6], especially in II, Section 2 (eqns (1)-(12)) and III, Section 3 (eqns (1)(4)). Matrix-oriented results are available in Gantmacher [7] and Piccinini et al [12]. Lyapunov observed (see [7]) that if the matrices $M, C, K$ are periodic with the same period, then a state variable transformation can be defined so that the transformed system has constant matrices. For simplicity, the assumption of constant (or piecewise constant) matrices is maintained henceforth.

Lagrangian systems can often be simplified by conversion to Hamiltonian form and transformation of the Hamiltonian variables by a contact transformation (Hamel [8]). This has not been effective for general dissipative systems, though there are interesting exceptions (Toda [15]). Rayleigh [13] and Caughey [4] found that solutions of Rayleigh systems could be expressed in terms of solutions of non-dissipative systems under special assumptions on $C_{1}$, in terms of $M$ and $K_{1}$. This procedure is here called reduction.

Duncan [5] and Brandon [3] adjoined $M \dot{q}-M \dot{q}=0$ or $K \dot{q}-K \dot{q}=0$ to the special Rayleigh system $M \ddot{q}+C_{1} \dot{q}+K_{1} q=f$ to obtain a symmetric first-order system in the state vector $[q, \dot{q}]$, which is equivalent to a nondissipative system. No special assumption on $C_{1}$ is required, but $C_{2}=$ $K_{2}=0$ in their approach.

In the analysis of large flexible systems subject to possible gyroscopic or circulatory forces, none of the methods described above is quite appropriate. The possibility of simplification of a general Rayleigh system by a state variable transformation is suggested by the successful use in mechanics of action-angle variables and the use in quantum mechanics and electromagnetism of complex combinations of position and momentum. These simple devices reduce apparently intractable systems into rather accessible forms.

Many choices will appear for the desired transformation, which have similar physical meaning but are sharply different from the viewpoint of com- 
putational efficiency. Some involve pairs of matrix inversions, and others involve solutions of auxillary equations of the Lyapunov type, such as $A B+B A^{T}=C$, where $A A^{T}=M$. Matrix factorisations appear at several stages of the development, both for theoretical and computational reasons. The resulting compromises are not explored in depth in the present paper, as they certainly require numerical experiments.

\section{Classical results}

The general Rayleigh system is written

$$
M \ddot{q}+C \dot{q}+K q=f
$$

where $C=C_{1}+C_{2}, K=K_{1}+K_{2}$ as in Section 1. Dots indicate derivatives, as usual. Rayleigh [13] noted that the "proportional damping" hypothesis

$$
C_{1}=\alpha M+\beta K_{1}, \quad C_{2}=K_{2}=0
$$

permits reduction of the system (2.1) to a single generalised eigenproblem $\left(K_{1}-\nu M\right) \underline{y}=0$. The condition (2.2) was relaxed to a semi-commutation or intertwining condition

$$
\Delta=C_{1} M^{-1} K_{1}-K_{1} M^{-1} C_{1}=0, \quad C_{2}=K_{2}=0
$$

by Caughey [4]. From the perspective of this paper, (2.3) is better written in the equivalent form

$$
\left(Y C_{1} Y^{\top}\right)\left(Y K_{1} Y^{\top}\right)=\left(Y K_{1} Y^{\top}\right)\left(Y C_{1} Y^{\top}\right)
$$

where $Y=A^{-1}$ and $A A^{\top}=M$. Since $C_{1}, K_{1}$ are symmetric, they have complete eigensystems and so $Y C_{1} Y^{\top}$ and $Y K_{1} Y^{\top}$ have a common set of eigenvectors. Thus $\left(K_{1}-\delta M\right) y=0$ and $\left(C_{1}-\eta M\right) y=0$ have common eigenvectors, used in solving (2.1). Clearly

$$
\Delta=\left(\alpha M+\beta K_{1}\right) M^{-1} K_{1}-K_{1} M^{-1}\left(\alpha M+\beta K_{1}\right)=0,
$$

when (2.2) holds, so that (2.3) or (2.4) is valid.

If the special Rayleigh system $M \ddot{q}+C_{1} \dot{q}+K_{1} q=f$ is augmented by $M \dot{q}-M \dot{q}=0$, as suggested by Duncan [5], then the first order system

$$
\left[\begin{array}{cc}
0 & M \\
M & C_{1}
\end{array}\right]\left[\begin{array}{l}
\dot{q} \\
q
\end{array}\right]^{\cdot}+\left[\begin{array}{cc}
-M & 0 \\
0 & K_{1}
\end{array}\right]\left[\begin{array}{l}
\dot{q} \\
q
\end{array}\right]=\left[\begin{array}{l}
0 \\
f
\end{array}\right]
$$

results. Note that both matrices which appear in (2.5) are symmetric, so that the associated generalised irregular eigenproblem

$$
\left(\left[\begin{array}{cc}
-M & 0 \\
0 & K_{1}
\end{array}\right]-\lambda\left[\begin{array}{cc}
0 & M \\
M & C_{1}
\end{array}\right]\right) y=0
$$


is of familiar type. When the matrices $M, C_{1}, K_{1}$ are large and sparse the computational status of the related regular eigenproblem

$$
\left(\left[\begin{array}{cc}
-M & 0 \\
0 & K_{1}
\end{array}\right]-\lambda\left[\begin{array}{cc}
\varepsilon I & M \\
M & C_{1}
\end{array}\right]\right) y=0
$$

can be assessed by many methods (Stewart [14]). Note that the regular form involves $M,-M-\varepsilon \cdot \lambda I$, and the matrix pencil $K_{1}-\lambda C_{1}$.

The alternative augmentation by $-K_{1} \dot{q}+K_{1} \dot{q}=0$, due to Brandon [3] yields

$$
\left[\begin{array}{cc}
M & 0 \\
0 & -K_{1}
\end{array}\right]\left[\begin{array}{l}
\dot{q} \\
q
\end{array}\right]+\left[\begin{array}{cc}
C_{1} & K_{1} \\
K_{1} & 0
\end{array}\right]\left[\begin{array}{l}
\dot{q} \\
q
\end{array}\right]=\left[\begin{array}{l}
f \\
0
\end{array}\right]
$$

and an associated symmetric eigenproblem

$$
\left(\left[\begin{array}{cc}
C_{1} & K_{1} \\
K_{1} & 0
\end{array}\right]-\lambda\left[\begin{array}{cc}
M & 0 \\
0 & -K_{1}
\end{array}\right]\right) y=0
$$

This at least is regular, and involves the matrix pencil $C_{1}-\lambda M$ and the matrix $K_{1}$, which may be preferable to the pairing $M$ and $K_{1}-\lambda C_{1}$ of Duncan. The method suggested below uses ideas drawn from control theory, the Caughey method, the Brandon method, and known state variable transformations of mechanics.

\section{Some state variable transforms}

In classical dynamics, quadratic Hamiltonians of the diagonalised form $H=\frac{1}{2} \sum_{k}\left(q_{k}^{2}+\dot{q}_{k}^{2}\right)$ are transformed by the action-angle variables $\tan \theta_{k}=$ $q_{k} / \dot{q}_{k}, q_{k}^{2}+\dot{q}_{k}^{2}=\omega_{k} J_{k}$, where $\dot{J}_{k}=0$ and $\dot{\theta}_{k}=\omega_{k}$ are the transformed equations. Alternatively, the complex quantities

$$
\omega_{k}=\dot{q}_{k}+i q_{k}, \quad \omega_{k}^{*}=\dot{q}_{k}-i q_{k}
$$

can be used as state variables, yielding $H=\frac{1}{2} \sum_{k} \omega_{k}^{*} \omega_{k}$. Similarly, in quantum mechanics (Landau [9]), if

$$
H(q, \dot{q})=\left(\dot{q}^{\top} M \dot{q}+q^{\top} K q\right) / 2
$$

the choice

$$
w=P \dot{q}+i Q q, \quad w^{*}=P \dot{q}-i Q q
$$

leads to the expression

$$
H=\left(w^{\top}\right)^{*} w / 2=\left(\dot{q}^{\top} P^{\top} P \dot{q}+q^{\top} Q^{\top} Q q\right) / 2
$$

with corresponding simplifications of the quantum "equations of motion" in the Heisenberg representation. Thus

$$
P^{\top} P=M, \quad Q^{\top} Q=K
$$


Here the vectors $q, \dot{q}$ are formally infinite.

Now, in the Rayleigh system

$$
M \ddot{q}+C \dot{q}+K q=f
$$

consider the linear state transformation

$$
w=A_{3} \dot{q}+B_{3} q
$$

and a corresponding equation of motion

$$
A_{4} \dot{w}+B_{4} w+F q=f .
$$

The use of five conditioning matrices $A_{3}, B_{3}, A_{4}, B_{4}, F$ in formulating the state-space equations simultaneously generalises the devices of Rayleigh, Hamel, Duncan, Caughey and Brandon in mechanics, Lyapunov in stability and control theory, and Landau in quantum mechanics. This innovation provides maximum flexibility in choosing the state space, while keeping the resulting calculations well within existing theoretical and computational resources. Once this expanded approach is adopted, many new results are obtained with moderate effort. Since $A_{4}\left(A_{3} \dot{q}+B_{3} q\right)^{\circ}+B_{4}\left(A_{3} \dot{q}+B_{3} q\right)+F q=f$ from (3.7) and (3.8), comparison with (3.6) yields

$$
A_{4} A_{3}=M, A_{4} B_{3}+B_{4} A_{3}=C, B_{4} B_{3}+F=K
$$

Note the similarity with (3.5), when $C=F=0$.

The set (3.9) of three bilinear matrix equations for the five state-space conditioning matrices has several unique characteristics. Firstly, it allows for two effectively arbitrary conditioning matrices to be chosen, potentially off-setting difficulties due to failures of the Rayleigh or Caughey conditions to hold between the mechanical (or electrical) system matrices $M, C, K$. Secondly, the equation $A_{4} A_{3}+B_{4} A_{3}=C$ which is key to the above system, is a wide generalisation of the Lyapunov condition (for constructing a Lyapunov function to test for stability of a linear system). The extensive material in the Russian and other literatures on the Lyapunov equation, and on the related Sylvester equation, permits efficient exploitation of the new approach.

Solving (3.9) first for $A_{4}$ and $A_{3}$, then for $B_{4}$ and $B_{3}$, and last for $F$, there are effectively two equations in four matrix unknowns. Each solution produces a different state space, and has a different effect on computing. This underscores the artificiality of state space representations, but does not spoil their usefulness.

The first problem is solving $A_{4} A_{3}=M$. Five solutions suggest themselves. The first choice is

$$
A_{4}=A_{3}=A=M^{1 / 2}
$$


where $M^{1 / 2}$ is the unique positive definite symmetric square root of $M$. More generally, $A_{4}=\alpha M^{1 / 2}, A_{3}=\alpha^{-1} M^{1 / 2}$ may have advantages, where the scalar $\alpha$ is suitably chosen.

The second choice (or set of choices) is

$$
A_{4}=L D_{4}, \quad A_{3}=D_{3} L^{T}
$$

where $L$ is a lower triangular matrix with $l$ 's on the diagonal, $D_{4}$ and $D_{3}$ are diagonal,

$$
A_{4} A_{3}=L D L^{\top}=M, \quad D=D_{4} D_{3}
$$

is the (Gauss) triangular factorisation of $M$, called the $L D U$ decomposition. Three subchoices for $D_{4}$ and $D_{3}$, and several detailed algorithms are available. The first subchoice is to take $D_{4}=I$ and to put the pivots $\left(p_{1}, p_{2}, \ldots\right)$ in $D_{3}$. The second subchoice is to take $D_{3}=I$ and to put the pivots in $D_{4}$. The third subchoice, due to Cholesky, is to put $D_{3}=D_{4}=\operatorname{diag}\left(p_{1}^{1 / 2}, p_{2}^{1 / 2}, \ldots\right)$, so that $A_{3}=A_{4}^{\top}$. Since $M$ is positive definite, $D_{3}=D_{4}$ is well-defined in spite of the square roots. Evidence exists that the Cholesky variant is more stable numerically, when the mass matrix $M$ has a mixture of small and large entries, than the other two variants and is only slightly slower.

The third choice is

$$
A_{4}=A=U \Sigma^{1 / 2}, \quad A_{3}=A^{\top}=\Sigma^{1 / 2} U^{\top}
$$

where $U$ is orthogonal and $\Sigma$ is the diagonal matrix of the eigenvalues of $A A^{T}=M$. Both (3.10) and (3.12) can be determined from the eigenvalues and eigenvectors of $M$.

Thus $A_{4} A_{3}=M=U \Sigma^{\top}$, a formula known as the singular value decomposition, where $\Sigma^{1 / 2}$ is the singular value matrix.

The fourth choice is

$$
A_{4}=V S_{1}, \quad A_{3}=S_{2} V^{\top}
$$

where $M=V S_{1} S_{2} V^{\top}=V R V^{\top}, V$ is orthogonal and $S_{1} S_{2}=R$ is upper quasi-triangular (that is, upper triangular except for possible $2 \times 2$ blocks along the diagonal). Generally, $S_{1}$ and $S_{2}$ satisfying $S_{1} S_{2}=R$ can be found which are also upper quasi-triangular, besides the trivial choice $S_{1}=$ $I, S_{2}=R$.

The fifth choice, rather like the fourth choice, is

$$
A_{4}=W H_{1}, \quad A_{3}=H_{2} W^{\top}
$$

where $M=W H_{1} H_{2} W^{T}=W J W^{T}, W$ is orthogonal and $H_{1} H_{2}=J$ is upper Hessenberg (upper triangular plus one diagonal below the main diagonal). 
Generally, $H_{1}$ and $H_{2}$ satisfying $H_{1} H_{2}=J$ can be found so that $H_{1}$ is upper quasi-triangular and $\mathrm{H}_{2}$ is also upper Hessenberg. This construction oddly enough is much faster computationally than the preceding one. Note that in each of the first three choices $A_{3}=A_{4}^{\top}$, adding to the symmetry of the solution. However, the unsymmetrical factorisations involved in the fourth and fifth choices may reduce the number of flops (combined multiplication and addition steps) required, not only in the first equation but also in the second equations discussed below.

The second problem is to solve $A_{4} A_{3}+B_{4} A_{3}=C$, where $A_{3}$ and $A_{4}$ have been determined to solve $A_{4} A_{3}=M$, for example by any of the five choices of $A_{3}$ and $A_{4}$ outlined above.

Note that $A_{4}$ and $-A_{3}$ will have no eigenvalues in common, since $M=$ $A_{4} A_{3}$ is symmetric positive definite and so has all positive eigenvalues. This is important to solve the second equation for the choice $B_{3}=B_{4}=B$, since the linear mapping $L^{\prime}(B)=A_{4} B+B A_{3}$ is nonsingular under the above eigenvalue condition on $A_{4}$ and $-A_{3}$.

The most direct approach to the second problem is through the pair of equations

$$
A_{4} B_{3}=C_{1}, \quad B_{4} A_{3}=C_{2}
$$

where $C_{1}, C_{2}$ are parts of $C$. Clearly, this will satisfy the second equation. The solution is

$$
B_{3}=A_{4}^{-1} C_{1}, \quad B_{4}=C_{2} A_{3}^{-1}
$$

and $F=K-B_{4} B_{3}=K-C_{2} A_{3}^{-1} A_{4}^{-1} C_{1}=K-C_{2} M^{-1} C_{1}$. Thus $F$ is independent of the various choices of $A_{3}$ and $A_{4}$ when this route is chosen. Also, $F=K$ if $C_{1}=0$ or $C_{2}=0$, which explains why these cases do simplify the reduction process. Some numerical evidence indicates that a less direct method is more efficient.

Before discussing the Lyapunov-type choice $B_{3}=B_{4}=B$, it will be helpful to examine the new state space equations in the first order evolutionary form

$$
\left[\begin{array}{cc}
A_{4} & 0 \\
0 & A_{3}
\end{array}\right]\left[\begin{array}{c}
w \\
q
\end{array}\right]^{\circ}+\left[\begin{array}{cc}
B_{4} & K-B_{4} B_{3} \\
-I_{n} & B_{3}
\end{array}\right]\left[\begin{array}{l}
w \\
q
\end{array}\right]=\left[\begin{array}{l}
f \\
0
\end{array}\right]
$$

This vector equation is the desired general state-space form of the original Rayleigh equation and all further developments here center on elucidation of (3.17).

The analysis of (3.17) simplifies when the $(2 n) \times(2 n)$ evolution matrix

$$
G=\left[\begin{array}{cc}
B_{4} & K-B_{4} B_{3} \\
-I_{n} & B_{3}
\end{array}\right]
$$


is factored into reducible matrices. More generally, consider

LEMMA 1.

$$
G-\lambda I_{2 n}=\left[\begin{array}{cc}
K_{\lambda} & \lambda I_{n}-B_{4} \\
0 & I_{n}
\end{array}\right]\left[\begin{array}{cc}
0 & I_{n} \\
-I_{n} & 0
\end{array}\right]\left[\begin{array}{cc}
I_{n} & \lambda I_{n}-B_{3} \\
0 & I_{n}
\end{array}\right]
$$

where

$$
K_{\lambda}=K-\lambda\left(B_{3}+B_{4}\right)+\lambda^{2} I_{n}
$$

Proof. Multiplication of the last two partitioned matrices yields

$$
\left[\begin{array}{cc}
0 & I_{n} \\
-I_{n} & B_{3}-\lambda I_{n}
\end{array}\right]
$$

Further multiplication leads to

$$
\begin{aligned}
& {\left[\begin{array}{cc}
B_{4}-\lambda I_{n} & K_{\lambda}-\left(B_{3}-\lambda I_{n}\right)\left(B_{4}-\lambda I_{n}\right) \\
-I_{n} & B_{3}-\lambda I_{n}
\end{array}\right]} \\
& \quad=\left[\begin{array}{cc}
B_{4} & K-B_{3} B_{4} \\
-I_{n} & B_{3}
\end{array}\right]-\lambda I_{2 n}=G-\lambda I_{2 n}
\end{aligned}
$$

LEMMA 2.

$$
\operatorname{det}\left(G-\lambda I_{2 n}\right)=\operatorname{det}\left(K_{\lambda}\right)
$$

Proof. Since the factors in Lemma 1 are all reducible with at least one $n$ by $n$ block of zeros, $\operatorname{det}\left(G-\lambda I_{2 n}\right)=\operatorname{det}\left(K_{\lambda}\right) \cdot 1$.

THEOREM 1. The evolution matrix $G$ of system (3.19) has the following properties (i) The eigenvalues of $G$ are the zeros of $\operatorname{det}\left(K_{\lambda}\right)$. (ii) The right eigenvectors of $G$ are determined by the eigenvectors of $B_{3}$ and the right null vectors of $K_{\lambda}$, as shown below. (iii) The left eigenvectors of $G$ are determined by $B_{4}$ and the left null vectors of $K_{\lambda}$, as shown below.

Proof. (i) is settled by Lemma 2. As for (ii), if $v$ is a column vector $\left[\begin{array}{l}v^{(1)} \\ v^{(2)}\end{array}\right]$, then by Lemma 1 ,

$$
\left(G-\lambda I_{2 n}\right) v=\left[\begin{array}{cc}
K_{\lambda} & \lambda I_{n}-B_{4} \\
0 & I_{n}
\end{array}\right]\left[\begin{array}{c}
v^{(2)} \\
\left(B_{3}-\lambda I_{n}\right) v^{(2)}-v^{(1)}
\end{array}\right]
$$

Hence $v$ is a right eigenvector if one of four possibilities occurs:

(a) $v^{(2)}=0$ and $\left(B_{3}-\lambda I_{n}\right) v^{(2)}-v^{(1)}=0$, that is $v^{(1)}=0$ which is a trivial case; or,

(b) $v^{(2)}=0$ and $v^{(1)} \neq 0$, while on multiplying, $\left(\lambda I_{n}-B_{4}\right) v^{(1)}=$ 0 , and $v^{(1)}=0$, a contradiction; or 
(c) $v^{(2)} \neq 0$ and $\left(B_{3}-\lambda I_{n}\right) v^{(2)}-v^{(1)}=v^{(3)}=0, K_{\lambda} v^{(2)}=0$, so $v^{(2)}$ is a right null vector of $K_{\lambda}$ and $v^{(1)}=\left(B_{3}-\lambda I_{n}\right) v^{(2)}$;

(d) $v^{(2)} \neq 0, v^{(3)} \neq 0$, while on multiplying out $K_{\lambda} v^{(2)}+\left(\lambda I_{n}-B_{4}\right) v^{(3)}=0$ and $v^{(3)}=0$, another contradiction.

As for (iii), suppose that $\left[w^{(1)}, w^{(2)}\right]\left(G-\lambda I_{2 n}\right)=0$, so by Lemma 1 ,

$$
\begin{aligned}
& {\left[w^{(1)} K_{\lambda}, w^{(1)}\left(\lambda I_{n}-B_{4}\right)+w^{(2)}\right]\left[\begin{array}{cc}
0 & I_{n} \\
-I_{n} B_{3}-\lambda & I_{n}
\end{array}\right]} \\
& \quad=\left[w^{(1)}\left(B_{4}-\lambda I_{n}\right)-w^{(2)}, w^{(1)}\left(K-B_{4} B_{3}\right)+w^{(2)}\left(B_{3}-\lambda I_{n}\right)\right]=[0,0]
\end{aligned}
$$

Hence $w$ is a left eigenvector whenever

$$
w^{(2)}=w^{(1)}\left(B_{4}-\lambda I_{n}\right) \text { and } w^{(2)}\left(B_{3}-\lambda I_{n}\right)=w^{(1)}\left(B_{4} B_{3}-K\right),
$$

that is, whenever

$$
w^{(1)}\left[\left(B_{4}-\lambda I_{n}\right)\left(B_{3}-\lambda I_{n}\right)+K-B_{4} B_{3}\right]=w^{(1)} K_{\lambda}=0 .
$$

That means $w^{(1)}$ is a left null vector of $K_{\lambda}$ and $w^{(2)}$ is determined by $w^{(1)}$ and $B_{4}$. This is another way of looking at $\operatorname{det} K_{\lambda}=0$. Note that if

$$
K\left(B_{3}+B_{4}\right)=\left(B_{3}+B_{4}\right) K
$$

where $K$ and $B_{3}+B_{4}$ are assumed to have complete sets of eigenvectors, then the left eigenvectors of $G$ are determined by $K$, by reasoning around (2.4).

THEOREM 2. If $N$ is an intertwining operator for $K$ and $\left(B_{3}+B_{4}\right)$, then $K$ and $N$ primarily determine $K_{\lambda}$.

Proof. The intertwining property

$$
K N\left(B_{3}+B_{4}\right)=\left(B_{3}+B_{4}\right) N K
$$

can be exploited as follows. Let $N=U \Sigma V^{*}$ where $U$ and $V$ are unitary and $\Sigma$ is diagonal ( $S V$ decomposition). Now let $\widehat{K}=\Sigma^{1 / 2} V^{*} K U \Sigma^{1 / 2}, \quad B_{5}=B_{3}+B_{4}, \quad \widehat{B}_{5}=\Sigma^{1 / 2} V^{*}\left(B_{3}+B_{4}\right) U \Sigma^{1 / 2}$, etc.

Then

$$
\begin{aligned}
\widehat{K} \widehat{B}_{5} & =\Sigma^{1 / 2} V^{*} K U \Sigma^{1 / 2} \Sigma^{1 / 2} V^{*}\left(B_{3}+B_{4}\right) U \Sigma^{1 / 2} \\
& =\Sigma^{1 / 2} V^{*} K N\left(B_{3}+B_{4}\right) U \Sigma^{1 / 2} \\
& =\Sigma^{1 / 2} V^{*}\left(B_{3}+B_{4}\right) N K U \Sigma^{1 / 2} \\
& =\Sigma^{1 / 2} V^{*}\left(B_{3}+B_{4}\right) U \Sigma^{1 / 2} \Sigma^{1 / 2} V^{*} K U \Sigma^{1 / 2} \\
& =\widehat{B}_{5} \widehat{K} .
\end{aligned}
$$


Thus if $\widehat{B}_{5}$ and $\widehat{K}$ have simple eigenvalues $\left\{\gamma_{j}\right\},\left\{\kappa_{j}\right\}$ (which we assume) and $\widehat{K} x_{j}=\kappa_{j} x_{j}$, then $\widehat{B}_{5} x_{j}=\gamma_{j} x_{j}$ and so

$$
\begin{aligned}
\widehat{K}_{\lambda} x_{j} & =\left(\Sigma^{1 / 2} V^{*} K_{\lambda} U \Sigma^{1 / 2}\right) x_{j} \\
& =\kappa_{j} x_{j}-\lambda \gamma_{j} x_{j}+\Sigma^{1 / 2} V^{*} \lambda^{2} U \Sigma^{1 / 2} x_{j} \\
& =\left(E-\lambda \Gamma+\lambda^{2} \widehat{I}\right) x_{j}
\end{aligned}
$$

where $E=\operatorname{diag}\left(\kappa_{j}\right), \Gamma=\operatorname{diag}\left(\gamma_{j}\right)$, and $\widehat{I}=\Sigma^{1 / 2} V^{*} U \Sigma^{1 / 2}$. If $X$ is the eigenvector matrix $\left[x_{1}\left|x_{2}\right| \ldots\right]$ then $\left(K_{\lambda}-\lambda^{2} I\right)^{\wedge}=X(E-\lambda \Gamma) X^{-1}$ and so

$$
K_{\lambda}=\lambda^{2} I+V \Sigma^{-1 / 2} X(E-\lambda \Gamma) X^{-1} \Sigma^{-1 / 2} U^{*} .
$$

In this formula, $U, V, \Sigma$ are determined by $N$, while $X$ and $E$ are determined by $K$, and

determines $\Gamma$.

$$
\gamma_{j}=x_{j}^{*} \Sigma^{1 / 2} V^{*} B_{5} U \Sigma^{1 / 2} x_{j} /\left|x_{j}\right|^{2}
$$

If $A \times B$ denotes the Kronecker product of any two matrices $A$ and $B$, then (3.24) has a nontrivial solution $N$ if and only if

$$
\operatorname{det}\left[K \times\left(B_{3}+B_{4}\right)^{T}-\left(B_{3}+B_{4}\right) \times K^{T}\right]=0
$$

as follows from writing $N$ as a vector and (3.24) as a vector equation. The above "Kronecker difference" determinant is relatively simple, and can be evaluated in $n^{2}$ steps by the conjugate gradient method, but is not discussed further here. Condition (3.28) is the generalised substitute for the Caughey condition (2.3) - which in the present language says that $M^{-1}$ is an intertwining operator for $K_{1}$ and $C_{1}$. Since it is a single scalar condition, it is more easily satisfied than the $n(n+1) / 2$ scalar conditions of Caughey.

\section{Lyapunov-type transformations}

Another type of solution to the second equation of (3.9) is provided by the choice $B_{3}=B_{4}=B$, resulting in the equation

$$
A_{4} B+B A_{3}=C
$$

Strictly (4.1) is a Lyapunov equation only when $A_{3}=A_{4}^{T}$, but the solution methods are similar. The condition for (4.1) to have a solution for any $C$ is that

$$
\operatorname{det}\left(A_{4} \oplus A_{3}\right)=\operatorname{det}\left(A_{4} \times I+I \times A_{3}\right) \neq 0
$$

where $\oplus$ denotes Kronecker sum. Now (4.2) holds if and only if $A_{4}$ and $-A_{3}$ have no eigenvalues in common. Since $A_{4} A_{3}=M$ is positive definite, 
the real parts of the eigenvalues of $A_{3}$ and $A_{4}$ all have the same sign, which can be taken as positive, so that condition (4.2) is assured.

The construction of the solution of (4.1) can be accomplished in four quite different ways. The most elegant is Bellman's integral solution. There is also a formal infinite series, which can be condensed via characteristic equations to a finite expression. These three solutions have theoretical advantages, but unless $A_{4}$ and $A_{3}$ are in some way special, are not rapidly calculable. Finally, methods based on Gaussian (triangular, Schur, or Hessenberg) reduction algorithms are computationally efficient but theoretically opaque. These methods interact with five types of choices made for $A_{3}$ and $A_{4}$ to a significant extent, as far as the number of required work steps of combined addition and multiplication are concerned.

Bellman's solution [2] is

$$
B=B_{1}+B_{2}, \quad B_{j}=\int_{0}^{\infty} \exp \left(-A_{4} t\right) C_{j} \exp \left(-A_{3} t\right) d t
$$

where $C=C_{1}+C_{2}, C_{1}$ is symmetric, $C_{2}$ is anti-symmetric, and $t$ has physical dimension (mass) ${ }^{1 / 2}$. If $A_{3}=A_{4}^{\top}$, as in the first three choices of $A_{3}, A_{4}$ made in Section 3, then $B_{1}^{T}=B_{1}$ and $B_{2}^{\top}=-B_{2}$.

Also if $A_{4}$ is triangular (lower or upper), then $\exp \left(-A_{4} t\right)$ is also triangular, and similarly for $A_{3}$ and $\exp \left(-A_{3} t\right)$, since powers of triangular matrices are triangular. Thus if $C=C_{1}+C_{2}$ has a triangular $(L D U)$ decomposition, $C=L C_{0} U$, then each matrix $\exp \left(-A_{4} t\right) C \exp \left(-A_{3} t\right)$ has a known $L D U$ decomposition.

With the first choice $A_{3}=A_{4}=M^{1 / 2}$, an explicit formula for $B$ is possible. Let $p_{j}, j=1, \ldots, n$ be the orthonormal eigenvectors of $M$, and $m_{j j}$ be the corresponding eigenvalues, so that

$$
M^{1 / 2}=\sum_{j} m_{j j}^{1 / 2} p_{j} p_{j}^{\top}
$$

and

$$
\exp \left(-M^{1 / 2} t\right)=\sum_{j} \exp \left(-m_{j j}^{1 / 2} t\right) p_{j} p_{j}^{\top}
$$

If

$$
\gamma_{j k}=p_{j}^{T} C p_{k}, \quad C=\sum_{j, k} \gamma_{j k} p_{j} p_{k}^{\top}
$$

is the orthogonal decomposition of $C$ into the dyads $p_{j} p_{k}^{\top}$ generated by $M$, then insertion of (4.5) and (4.6) into (4.3) yields

$$
B=\sum_{j, k} \beta_{j k} p_{j} p_{k}^{\top}, \quad \beta_{j k}=\gamma_{j k}\left(m_{j j}^{1 / 2}+m_{k k}^{1 / 2}\right)^{-1}
$$


upon term-by-term integration of (4.3).

The formal series for $B$ mentioned above is

$$
B=\sum_{m=0}^{\infty}\left(-A_{4}\right)^{m} C A_{3}^{-(m+1)} .
$$

Then $A_{4} B+B A_{3}=C+A_{4} C A_{3}^{-1}-A_{4} C A_{3}^{-1}+\cdots=C$. The dual series $B=\sum_{m=0}^{\infty} A_{4}^{-(m+1)} C\left(-A_{3}\right)^{m}$ also satisfies (4.1) formally. As for convergence, if \|\| denotes a matrix norm, then (4.8) converges if $\left\|A_{4}^{m} C A_{3}^{-m}\right\|<$ 1. But that is implied by $\|C\|\left(\left\|A_{4}\right\|\left\|A_{3}^{-1}\right\|\right)^{m}<1$ for all $m$ sufficiently large, and hence by the inequality $\rho=\left\|A_{4}\right\|\left\|A_{3}^{-1}\right\|<1$. The choice $A_{4}=\alpha M^{1 / 2}, A_{3}=\alpha^{-1} M^{1 / 2}$ yields $\rho=|\alpha|^{2}\left\|M^{1 / 2}\right\|\left\|M^{-1 / 2}\right\|=|\alpha|^{2} n\left(M^{1 / 2}\right)$, where $n(D)=\|D\|\left\|D^{-1}\right\|$ is the condition number of a matrix $D$. Hence $|\alpha|<\left(n\left(M^{1 / 2}\right)\right)^{-1 / 2}$ will guarantee convergence of (4.8).

Infinite series can be avoided by using the Hamilton-Cayley equations of $A_{3}$ and $A_{4}$ to condense (4.8) into a finite expression. This is primarily of theoretical interest, (Leipnik [11]), as it, like Bellman's formula is rather slow, compared to the methods of Bartels [1] or Van Loan [17] based on matrix factorization.

Briefly, the idea of Bartels is to use the Schur decompositions

$$
U_{4}^{\top} A_{4} U_{4}=R_{4} \text { and } U_{3}^{\top} A_{3}^{\top} U_{3}=R_{3}
$$

where $R_{4}$ and $R_{3}$ are upper or lower quasi-triangular and $U_{3}, U_{4}$ are orthogonal. Of course, if $A_{3}$ and $A_{4}$ have been chosen to be upper and lower triangular (as in (3.11)), or essentially unitary (as in (3.12)) or products of unitary and triangular (or unitary and Hessenberg) matrices, this task is eased.

The resulting equation based on (4.1) and (4.9) is

$$
R_{4} Y+Y R_{3}^{T}=Z
$$

where $Y=U_{4}^{\top} B U_{3}$ and $Z=U_{4}^{\top} C U_{3}$. The columns of $Y$ are expressible in terms of columns of higher index to produce an upper quasi-triangular system, requiring $10 n^{3}$ flops for $R_{4}$, another $10 n^{3}$ flops for $R_{3}^{\top}$ (if they are not simply related), and $5 n$ flops for the solution of (4.10). Also $2 n^{2}\left(+2 n^{2}\right)+$ $n^{2}$ storage locations are needed.

Van Loan's improvement is to relax one of the two reductions to be upper Hessenberg instead of upper quasi-triangular. Suppose then that $R_{4}$ is upper Hessenberg. This step now requires (Golub et al [17]) only $5 n^{3} / 3$ operations, using symmetric projections (Householder reflections). The equation (4.10) now requires $7.5 n^{3}$ flops, and storage locations totaling $6 n^{2}$. Hence about $19 n^{3}$ flops are required, a $25 \%$ reduction in work, with a $20 \%$ increase in storage (which is comparatively minor). 
A research project covering the combined computational effectiveness of the 15 proposed different methods ( 5 for the determination of the $A^{\prime} S$ and 3 for the determination of $B$ ) as applied to a set of typical Rayleigh problems with variable (but high) dissipations and/or nonzero gyroscopic forces $\left(C_{2} \neq\right.$ 0 ) and/or circulatory forces $\left(K_{2} \neq 0\right)$, is awaiting funding. As mentioned in the introduction, the usual treatments of high order systems are restricted to small dissipations, without gyroscopic or circulatory (lift) contributions. The credible removal of these restrictions, unrealistic for modern spacecraft, will require considerable effort.

Supposing that $(4.1)$ has been solved somehow for $B$, the state space equations are now

$$
\left[\begin{array}{cc}
A_{4} & 0 \\
0 & A_{3}
\end{array}\right]\left[\begin{array}{l}
w \\
q
\end{array}\right]+G\left[\begin{array}{l}
w \\
q
\end{array}\right]=\left[\begin{array}{l}
f \\
0
\end{array}\right]
$$

where $G=\left[\begin{array}{cc}B & K-B^{2} \\ -I & B\end{array}\right]$ and

$$
\begin{aligned}
G-\lambda I & =\left[\begin{array}{cc}
B-\lambda I & K-B^{2} \\
-I & B-\lambda I
\end{array}\right] \\
& =\left[\begin{array}{cc}
K-2 \lambda B+\lambda^{2} I & \lambda I-B \\
0 & I
\end{array}\right]\left[\begin{array}{cc}
0 & I \\
-I & 0
\end{array}\right]\left[\begin{array}{cc}
I & \lambda I-B \\
0 & I
\end{array}\right] .
\end{aligned}
$$

Here $\lambda$ has physical dimension (mass) $)^{1 / 2} /$ time.

As in (3.20)-(3.28), and Lemmas 1, 2 and Theorem 1 of Section 3, the eigenvalues and eigenvectors of $G$ are controlled by $B$ and the null vectors of $K_{\lambda}$. If $B K=K B$ or more generally $B N K=K N B$ for some $N$, then the eigenbehavior of $G$ is controlled by $K$ and $N$, as in Theorem 2 of Section 3. These results are also new, reducing to Caughey's when $C$ and $K$ are symmetric and $M^{-1}$ is an intertwining operator for them.

The simplification of (4.11) or its generalisation (3.17) is more complete if $G$ and $\left[\left(A_{4}^{\top}, 0\right)^{\top},\left(0, A_{3}^{\top}\right)^{\top}\right]$ commute or, more generally, if there is a (partitioned) nonzero $2 n \times 2 n$ intertwining operator such that

$$
\left[\begin{array}{cc}
A_{4} & 0 \\
0 & A_{3}
\end{array}\right]\left[\begin{array}{cc}
S & T \\
U & V
\end{array}\right]\left[\begin{array}{cc}
B_{4} & F \\
-I & B_{3}
\end{array}\right]=\left[\begin{array}{cc}
B_{4} & F \\
-I & B_{3}
\end{array}\right]\left[\begin{array}{cc}
S & T \\
U & V
\end{array}\right]\left[\begin{array}{cc}
A_{4} & 0 \\
0 & A_{3}
\end{array}\right]
$$

where $F=K-B_{4} B_{3}$. The formal proof of this result resembles that of Theorem 2 , but is rather longer. Then some associated operators will commute, as detailed in (3.24)-(3.28) in the $n \times n$ case.

After writing out (4.13) as four linear matrix equations in $S, T, U, V$, rescaling all quantities to dimensionless form, and converting the equations to vector equations in the coefficients of $S, T, U, V$ via Kronecker products, the condition for nontrivial solutions becomes the rather cumbersome 
equation

$$
\operatorname{det}\left[\begin{array}{cccc}
a_{11} & a_{12} & a_{13} & 0 \\
a_{21} & a_{22} & 0 & a_{24} \\
a_{31} & 0 & a_{33} & a_{34} \\
0 & a_{42} & a_{43} & a_{44}
\end{array}\right]=0
$$

where $a_{11}=A_{4} \times B_{4}^{\top}-B_{4} \times A_{4}^{\top}, a_{12}=-A_{4} \times I, a_{13}=-F \times A_{4}^{\top}, a_{21}=$ $A_{4} \times F^{\top}, a_{22}=A_{4} \times B_{3}^{\top}-B_{4} \times A_{3}^{\top}, a_{24}=-F \times A_{3}^{\top}, a_{31}=I \times A_{4}^{\top}, a_{33}=$ $A_{3} \times B_{4}^{\top}-B_{3} \times A_{4}^{\top}, a_{34}=-A_{3} \times I, a_{42}=I \times A_{3}^{\top}, a_{43}=A_{3} \times F^{\top}$, and $a_{44}=A_{3} \times$ $B_{3}^{\top}-B_{3} \times A_{3}^{\top}$. The columns are obtained from the entries of $S, T, U, V$, and the rows are obtained from the equations for the four blocks, in the order $(1,1),(1,2),(2,1),(2,2)$, which seems to be most convenient. Again the formal proof is tedious but direct. As usual, this determinant can be calculated by a Gauss triangularisation or conjugate gradient method. Equation (4.14) is another generalisation and weakening of the Caughey condition (2.3). Of course it simplifies when $B_{3}=B_{4}=B$ or $A_{4}=A_{3}=M^{1 / 2}$. An attractive feature is that if $M$ or $C$ contain adjustable parameters, then these may be chosen to satisfy (4.14) and hence increase the degree of reduction.

\section{System reduction}

The original system has been rewritten as

$$
\left[\begin{array}{cc}
A_{4} & 0 \\
0 & A_{3}
\end{array}\right]\left[\begin{array}{l}
w \\
q
\end{array}\right]+\left[\begin{array}{cc}
B_{4} & K-B_{4} B_{3} \\
-I_{1} & B-3
\end{array}\right]\left[\begin{array}{l}
w \\
q
\end{array}\right]=\left[\begin{array}{l}
f \\
0
\end{array}\right]
$$

and the eigenstructure revealed, where $A_{4} A_{3}=M$ and $A_{4} B_{3}+A_{3} B_{4}=C$. It remains to study the solution for given $f$. Now when $C=0$, take $B_{3}=B_{4}=0$ and consider the related system

$$
\left[\begin{array}{cc}
A_{4} & 0 \\
0 & A_{3}
\end{array}\right]\left[\begin{array}{c}
w_{0} \\
q_{0}
\end{array}\right]+\left[\begin{array}{cc}
0 & K \\
-I & 0
\end{array}\right]\left[\begin{array}{c}
w_{0} \\
q_{0}
\end{array}\right]=\left[\begin{array}{l}
f_{0} \\
g_{0}
\end{array}\right]
$$

Here $w_{0}=A_{3} \dot{q}_{0}-g_{0}$ is a conventional momentum-type variable attached to the non-dissipative equation

$$
M \ddot{q}_{0}+K q_{0}=f_{0}+A_{4} \dot{g}_{0}
$$

whose solution is supposed known. If now $f_{0}$ is taken to be $f-\tilde{f}$, where $\tilde{f}$ is to be chosen later, the result of subtracting (5.1) from (3.17) is

$$
\left[\begin{array}{cc}
A_{4} & 0 \\
0 & A_{3}
\end{array}\right]\left[\begin{array}{c}
\tilde{w} \\
\tilde{q}
\end{array}\right]^{\cdot}+\left[\begin{array}{cc}
B_{4} & K-B_{4} B_{3} \\
-I & B_{3}
\end{array}\right]\left[\begin{array}{c}
\tilde{w} \\
\tilde{q}
\end{array}\right]=\left[\begin{array}{c}
\tilde{f}-B_{4} w_{0}+B_{4} B_{3} q_{0} \\
-g_{0}-B_{3} g_{0}
\end{array}\right]
$$

where

$$
\tilde{w}=w-w_{0}, \quad \tilde{q}=q-q_{0}
$$


All the simplifications proposed in Sections 3 and 4 for the left side of the system (5.3) are available, and will not be recapitulated. For the right side, two further alternative simplifications suggest themselves. The first is to choose

$$
\tilde{f}=g_{0}=0
$$

so that the non-dissipative equation (5.2) has the same driving function as did the original Rayleigh equation. This results in the driving function

$$
\left[\left(B_{3} q_{0}-w_{0}\right)^{\top} B_{4}^{\top},-q_{0}^{\top} B_{3}^{\top}\right]^{\top}
$$

for the perturbation equation (5.3).

The second choice is to take $\left[\tilde{f}-B_{4} w_{0}+B_{4} B_{3} q_{0},-g_{0}-B_{3} q_{0}\right]^{\top}$ orthogonal to some of the solutions of

$$
\left[\begin{array}{cc}
A_{4} & 0 \\
0 & A_{3}
\end{array}\right]\left[\begin{array}{c}
\dot{w}_{00} \\
\dot{q}_{00}
\end{array}\right]+\left[\begin{array}{cc}
0 & K \\
-I & 0
\end{array}\right]\left[\begin{array}{c}
w_{00} \\
q_{00}
\end{array}\right]=0
$$

The orthogonality would be relative to the inner product

$$
\left(\left[\phi_{1}, \psi_{1}\right]^{\top},\left[\phi_{2}, \psi_{2}\right]^{\top}\right)=\int_{a}^{b}\left[\phi_{1}^{\top}, \psi_{1}^{\top}\right]\left(G+G^{\top}\right)\left[\phi_{2}^{\top}, \psi_{2}^{\top}\right]^{\top} d t
$$

where $a, b$ are two "times" natural to the problem, such as times at which $w_{0}$ and $q_{0}$ are known.

This results in a differential equation with integral constraints, effectively a "Green's method", which needs further investigation. In either case, $q=$ $q_{0}+\tilde{q}$ and $w=w_{0}+\tilde{w}$, where $q_{0}, w_{0}$ are related to a non-dissipative problem and $\tilde{q}, \tilde{w}$ are determined by a simplified problem, driven by "known" functions. This simplified problem may be determined itself by a nondissipative or partly decoupled dissipative problem if a commutation or determinantal condition holds, as seen in Sections 3 and 4.

\section{Acknowledgements}

The author wishes to thank Dr. Charles Kenney of UCSB for advice and Professor Alan Laub for suggesting the problem of simplification of general Rayleigh systems, and for his patience.

The referee is thanked for requesting clarifications in the material now covered by Theorems 1 and 2, with consequent improvements.

Office of Naval Research Support under contract N-000-14-85-K-0SS3 is gratefully acknowledged. 


\section{References}

[1] R. H. Bartels and G. W. Stewart, "Solution of the matrix equation $A X+X B=C$ (Algorithm 826)", Commun. of Assoc. of Comp. Mach. 15 (1972) 820-826.

[2] R. Bellman, Introduction to Matrix Analysis (McGraw Hill, New York, 1960).

[3] J. A. Brandon, "Discussion of alternative Duncan formulations of the eigenproblem for the solution of non-classically, viscously damped linear systems", Trans ASME, Ser. E, J. of Applied Mechanics 51,4 (1985) 605-609.

[4] T. K. Caughey and M. E. J. O'Kelly, "Classical normal modes in damped linear systems", Trans ASME, Ser. E, J. of Applied Mechanics 32,3 (1965) 583-588.

[5] W. J. Duncan, et al, Elementary matrices and some applications to dynamics and differential equations (Macmillian, New York, 1946, reprint).

[6] P. Frank and R. von Mises, Partial differential equations of physics (Dover, New York, 1961, reprint).

[7] F. R. Gantmacher, Theory of matrices Vol. I and II (Chelsea, New York, 1959, reprint).

[8] G. Hamel, Theoretische Mechanik (Springer, Berlin, 1949).

[9] L. D. Landau, Course of theoretical physics - quantum mechanics (Pergamon Press, London, 1982).

[10] P. D. Lax, Non-linear differential equations in applied science (Elsevier, New York, 1983).

[11] R. B. Leipnik, "Extension of Newman's formula for the Sylvester equation to the generic case", Linear and Multilinear Algebra 12,4 (1982/83) 305-309.

[12] L. C. Piccinini, et al, Ordinary differential equations in $R^{n}$ (Springer, Berlin, 1984).

[13] J. W. S. Rayleigh, Theory of sound (Dover, New York, 1945, reprint).

[14] G. W. Stewart and I. S. Duff, "Practical comparisons of codes for the solution of sparse linear systems", in Sparse matrix computation symposium (eds. G.W. Stewart and I.S. Duff) (SIAM Press, Philadelphia, 1976).

[15] N. Toda, Theory of non-linear lattices (Springer, Berlin, 1, 1981).

[16] J. M. Vance and A. Sitchin, "Derivation of first order difference equations for dynamical systems by direct applications of Hamilton's principle”, Trans ASME, Ser. E, J. of Applied Mechanics 37,2 (1970) 276-278.

[17] C. F. van Loan and G. H. Golub, et al, "A Hessenberg-Schur method for the solution of $A X+X B=C$ ", SIAM J. on Num. Anal. 17 (1980) 883-893. 